\title{
PULSESHAPING IN UWB SYSTEMS USING SEMIDEFINITE PROGRAMMING WITH NON-CONSTANT UPPER BOUNDS
}

\author{
Christian R. Berger, Michael Eisenacher, Holger Jäkel, Friedrich Jondral \\ Institut für Nachrichtentechnik \\ Universität Karlsruhe (TH), Germany
}

\section{ABSTRACT}

A critical obstacle for Ultra Wideband (UWB) communications is conformity to restrictions set on the allowed interference with other wireless devices. Using trains of $N$ amplitude modulated basic pulses gives an FIR filter like design when disregarding the power spectral density (PSD) of the basic pulse. These leads to implementation losses and should be avoided. We quantify these losses and introduce an FIR filter design using semidefinite programming which can incorporate the basic pulse PSD by introducing non-constant upper bounds in the design. This leads to optimal designs and increases available signal power at no extra implementation complexity, just by choosing more optimal filter coefficients.

\section{INTRODUCTION}

Ultra Wideband (UWB) is a new technology for short range, high data rate wireless communication [1]; inherently occupying an extreme bandwidth, UWB technology will have to be implemented as an overlay scenario in order to coexist with other existing communication systems. To avoid interference, the Federal Communications Commission (FCC) has established guidelines [2], enabling research and implementation of first equipment by setting forth stringent regulations on the radiated energy. Due to this the spectral shape of UWB signals becomes an important implementation aspect, adhering to constraints and still maximizing available signal power, to enable the targeted high data rate applications.

Since the employed ultra-short pulses are generated with analog components, their spectral shape is not easy to design. Replacing the analog pulses with digital designs is prohibited by the huge bandwidth and resulting sampling rates. Using an FIR prefilter before the pulse generators, the spectral shape can be controlled. When assuming the pulse power spectral density (PSD) to be constant, the problem simplifies to a classic FIR filter design problem [3,4]. We will take a closer look at this simplification and show that assuming the pulse PSD to be constant leads to considerable losses in available signal power. When taking into account the particular shape of the pulse PSD, the problem can still be formulated as a FIR filter design, but the spectral constraints become non-constant which can not be handled by typical FIR filter designs. We will extend a semidefinite programming FIR filter design to include non-constant upper bounds to achieve a signal design which is adapted to the basic pulse PSD and renders optimal results in view of utilized signal power.

\section{Signal Model And Pulseshaping Problem}

\section{A. Signal Model}

Our signal model will be impulse radio (IR) with time hopping (TH) and binary pulse amplitude modulation (PAM). Ultrashort pulses are the building block of this transmission scheme; the basic pulse on the channel is $p(t)$, e.g., the Gaussian Monocycle [5], with the power $\int p^{2}(t) d t=\varepsilon$. One pulse is sent during each frame duration $T_{f}$. Each data symbol consist of $N_{f}$ pulses, resulting in a total symbol length $T_{s}=N_{f} T_{f}$. The signal model can be expressed in the following way:

$$
u(t)=\sum_{k} b_{k} \frac{1}{\sqrt{N_{f} \varepsilon}} \sum_{l=0}^{N_{f}-1} p\left(t-l T_{f}-k T_{s}-c_{l} T_{c}\right)
$$

where $b_{k}$ are the PAM symbols $\{-1,1\}$ for each bit, $T_{c}$ is the chip period and $c_{l}$ are the user-specific TH codes, with $c_{l} T_{c}<$ $T_{f} \forall l$.

The PSD can be calculated in a standard fashion to:

$$
\Phi_{u u}(f)=\frac{1}{T_{s} N_{f} \varepsilon}|P(f)|^{2}\left|\sum_{l=0}^{N_{f}-1} e^{j 2 \pi\left(-l T_{f}-c_{l} T_{c}\right) f}\right|^{2} .
$$

When assuming the TH code $c_{l}$ to be integer-valued, independent und uniformly distributed, this can be approximated as $[6,7]$ :

$$
\Phi_{u u}(f) \approx \frac{1}{T_{s} \varepsilon}|P(f)|^{2} .
$$

Thus, the PSD of the basic pulse $p(t)$ is crucial to the PSD of the complete UWB signal. Therefore, it is necessary to select a pulse with optimal spectral properties.

\section{B. Linear Pulseshaping Problem}

The basic pulses $p(t)$ used in UWB systems are created with analog RF components. Therefore, designing the pulse to comply with some specific demands like the FCC spectral mask is rather difficult. Basically only the pulse duration and amplitude can be controlled which corresponds to the bandwidth and power in the PSD. Different digitally created pulse shapes have been suggested [8], whereby those pulses have to be generated of digital samples. Since the pulses need bandwidths of several $\mathrm{GHz}$, sampling nanosecond length pulses is highly demanding.

Using transmit filters to adapt to spectral constraints is also difficult to implement, since analog filters would have to be used with an enormous bandwidth. Instead using an FIR filter like approach [3,4], each basic pulse is repeated $N$ times with arbitrary amplitudes, created by the pulse generators used 
already for modulation. This is equivalent to prefiltering the signal before using the basic pulse as a transmit filter.

$$
u(t)=\sum_{k} b_{k} \frac{1}{\sqrt{N_{f} \varepsilon}} \sum_{l=0}^{N_{f}-1} \delta\left(t-l T_{f}-k T_{s}-c_{l} T_{c}\right) * p(t)
$$

Now $p(t)$ includes the prefiltering with amplitudes $w_{n}$, which will be design parameters, and the pulses $q(t)$ created by the usual pulse generators which can be chosen by hardware constraints,

$$
p(t)=\sum_{n=0}^{N-1} w_{n} q(t-n T)=w(t) * q(t)
$$

whereby $w(t)=\sum_{n=0}^{N-1} w_{n} \delta(t-n T)$. Since the convolution is associative, it is equivalent to first use $w(t)$ as a prefilter and then $q(t)$ as the analog transmit filter. The PSD accordingly is,

$$
|P(f)|^{2}=\Phi_{w w}(f)|Q(f)|^{2}
$$

and $\Phi_{w w}(f)$ can be calculated as,

$\Phi_{w w}(f)=\left|\sum_{n=0}^{N-1} w_{n} e^{j 2 \pi n T f}\right|^{2}=r_{0}+2 \sum_{n=1}^{N-1} r_{n} \cos (2 \pi n T f)$,

with $r_{n}=\sum_{k=0}^{N-1-n} w_{k} w_{k+n}$ being the non-periodic autocorrelation sequence of the $w_{n}$.

To formulate the pulseshaping problem, an optimality criterion has to be chosen. The effective power usage ratio $\eta$ is defined as the ratio of achieved signal power to the maximum power possible within the frequency interval of interest $\mathcal{F}_{p}$, limited by the FCC spectral mask $S(f)$ :

$$
\eta=\frac{\int_{\mathcal{F}_{p}}|P(f)|^{2} d f}{\int_{\mathcal{F}_{p}} S(f) d f}
$$

The PSD $\Phi_{w w}(f)$ will be periodic in the additional design parameter $1 / T$, therefore we choose $\mathcal{F}_{p}$ to be $[0,1 / 2 T]$ since this is the interval we will be able to influence. Outside this interval we will assume $|Q(f)|^{2}$ to be small enough to attenuate the period repetitions of $\Phi_{w w}(f)$. Accordingly $T$ and $q(t)$ will have to be chosen jointly which we will discuss later in detail.

The pulseshaping problem can now be formulated with respect to the FCC spectral mask $S(f)$ and the effective power usage ratio:

$$
\max _{w_{n}} \eta \quad \text { subject to }|P(f)|^{2} \leq S(f) f \in \mathcal{F}_{p},
$$

i.e., maximizing the transmitted power while adhering to all spectral constraints. This is not a very practical problem formulation, because it puts constraints on infinitely many $f$ and is highly non-linear. A problem formulation linear dependent on the $r_{n}$ can be achieved when noticing that

$$
\eta=\frac{1}{P_{S}} \int_{\mathcal{F}_{p}}|P(f)|^{2} d f=r_{0} c_{0}+2 \sum_{n=1}^{N-1} r_{n} c_{n}
$$

with $c_{n}=\frac{1}{P_{S}} \int_{\mathcal{F}_{p}} \cos (2 \pi n T f)|Q(f)|^{2} d f$ being constants and $P_{S}=\int_{\mathcal{F}_{p}} S(f) d f$.

Under the additional constraint that $r_{n}$ are a valid autocorrelation sequence, which is equivalent to $\Phi_{w w}(f) \geq 0 \forall f$, we can write the new optimization problem as,

$$
\begin{aligned}
\max _{r_{n}} r_{0} c_{0}+ & 2 \sum_{n=0}^{N-1} r_{n} c_{n} \quad \text { subject to } \\
& |P(f)|^{2} \leq S(f), \Phi_{w w}(f) \geq 0 \quad f \in \mathcal{F}_{P}
\end{aligned}
$$

which is now linear with twice infinite many constraints. This would require sampling of the constraints and introduction of an additional relaxation to ensure compliancy for all $f \in \mathcal{F}_{P}$. Instead when approximating the $|Q(f)|^{2}$ as constant within $\mathcal{F}_{P},|P(f)|^{2} \approx \Phi_{w w}(f)$ we have only constraints on $\Phi_{w w}(f)$ which is the PSD of an FIR filter. Accordingly FIR design methods can be used to optimize $\Phi_{w w}(f)$, e.g., the Parks McClellan algorithm [3].

Instead we will use linear matrix inequality formulation derived for FIR filter design, which does not depend on equiripple design and guarantees global optimal solutions for convex problems [9]. The constraints on $\Phi_{w w}(f)$ can be expressed as linear constraints on the autocorrelation coefficients $r_{n}$ and some positive semidefinite matrices. Since positive semidefinite matrices form a convex set, all these problems are convex and represent symmetric cones. Using an optimization package for symmetric cones [10], they can be solved optimally [4]. The results for $T=0.0 \overline{3} \mathrm{~ns}$ can be seen in Fig. 1, performance can be seen for $\eta_{1}$ in Tab. 1 .

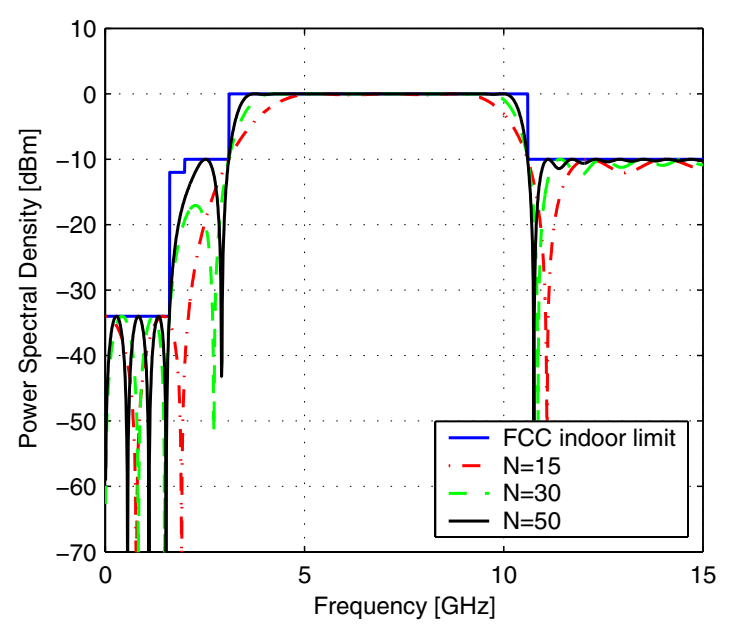

Figure 1: Optimized PSD assuming pulses with constant PSD

\section{Losses Due to Assuming a Constant Pulse PSD}

Assuming the pulse PSD $|Q(f)|^{2}$ to be flat over an area of easily $10 \mathrm{GHz}$ is a very strong simplification, even when using a pulse chosen to be as constant as possible at the frequencies of interest (see $|Q(f)|^{2}$ Fig. 2). When calculating the real PSD of the pulse $|P(f)|^{2}=\Phi_{w w}(f)|Q(f)|^{2}$, the multiplication with $|Q(f)|^{2}$ leads to considerable losses (see $\eta_{2}$ and losses in Tab. 
1 and Fig. 2). These losses are most noticeable when $\eta$ approaches unity for high lengths of $N$, but they will be much higher if the basic pulse $q(t)$ can not be freely chosen, e.g., due to hardware constraints, because any other pulse would be even further from the constant spectrum assumption.

\begin{tabular}{c|ccc}
$\mathrm{N}$ & 15 & 30 & 50 \\
\hline$\eta_{1}$ assuming constant pulse & 0.778 & 0.877 & 0.929 \\
\hline$\eta_{2}$ due to non-constant pulse & 0.633 & 0.698 & 0.726 \\
\hline losses [dB] & 0.899 & 0.989 & 1.073
\end{tabular}

Table 1: Performance assuming a constant pulse PSD and losses due to real pulse PSD

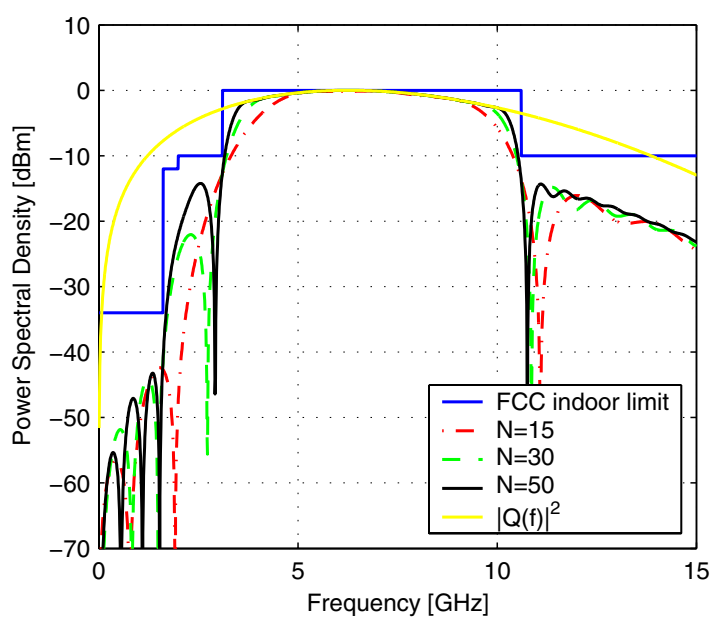

Figure 2: Losses in available signal power due to the nonnegligible basic pulse PSD

So a first evaluation of the simplification used in B. shows losses which increase with the optimality of the solution. Since this is contra productive to the goal of optimization, these losses have to be countered. This leads to a new pulseshaping problem.

\section{New Pulseshaping Problem}

\section{A. Formulation of New Pulseshaping Problem}

The original linear pulseshaping problem in B. needed to assume the PSD of the basic pulse as constant over a large interval. This was necessary to achieve a linear problem. To keep a linear problem formulation, but not neglect the pulse PSD a different approach can be taken,

$$
\begin{aligned}
& \max _{r_{n}} r_{0} c_{0}+2 \sum_{n=0}^{N-1} r_{n} c_{n} \quad \text { subject to } \\
& |P(f)|^{2} \leq \frac{S(f)}{|Q(f)|^{2}}, \Phi_{w w}(f) \geq 0 \quad f \in \mathcal{F}_{P} .
\end{aligned}
$$

Dividing the FCC spectral mask by the PSD of the Gaussian monocycle (see Fig. 3), as required in eq. (12), the optimization problem remains linear, but new constraints need to be implemented. These new constraints couldn't be implemented before, because the linear matrix inequalities borrowed from FIR filter design [9] can only express piecewise-constant constraints. Therefore, the linear matrix inequalities will have to be extended to cover more flexible constraints.

\section{B. Review of Linear Matrix Inequalities}

The linear matrix inequalities used before define the following positive cones:

$$
\begin{aligned}
& \mathcal{K}(\alpha)=\left\{\mathbf{p} \in \mathbb{R}^{n+1} \mid \sum_{k=0}^{n} p_{k} \cos (k \theta) \geq 0 \forall \theta \in[\alpha, \pi]\right\}, \\
& \overline{\mathcal{K}}(\alpha)=\left\{\mathbf{p} \in \mathbb{R}^{n+1} \mid \sum_{k=0}^{n} p_{k} \cos (k \theta) \geq 0 \forall \theta \in[0, \alpha]\right\} .
\end{aligned}
$$

Using the linear operators $\mathbf{L}^{*}: \mathbb{R}^{(n+1) \times(n+1)} \rightarrow \mathbb{R}^{n+1}$ and $\Lambda^{*}: \mathbb{R}^{n \times n} \rightarrow \mathbb{R}^{n+1}$ [9, eqs. (35)-(36)], it can be shown [9] that eqs. (13) and (14) are equivalent to:

$$
\begin{array}{r}
\mathcal{K}(\alpha)=\left\{\mathbf{p} \in \mathbb{R}^{n+1} \mid \mathbf{p}=\mathbf{L}^{*}(\mathbf{X})+\mathbf{\Lambda}^{*}(\mathbf{Z}, \alpha, 2 \pi-\alpha)\right. \\
\text { for some } \left.\mathbf{X} \in \mathcal{S}_{+}^{(n+1) \times(n+1)}, \mathbf{Z} \in \mathcal{S}_{+}^{n \times n}\right\} \\
\overline{\mathcal{K}}(\alpha)=\left\{\mathbf{p} \in \mathbb{R}^{n+1} \mid \mathbf{p}=\mathbf{L}^{*}(\mathbf{X})-\mathbf{\Lambda}^{*}(\mathbf{Z}, \alpha, 2 \pi-\alpha)\right. \\
\text { for some } \left.\mathbf{X} \in \mathcal{S}_{+}^{(n+1) \times(n+1)}, \mathbf{Z} \in \mathcal{S}_{+}^{n \times n}\right\}
\end{array}
$$

These positive cones can be used to implement piecewise constant upper bounds on $\Phi_{w w}(f)$. For example, the equality between eqs. (13) and (15) results to $\Phi_{w w}(f) \leq \gamma$ for $f \in$ $\left[\frac{\alpha}{2 \pi T}, \frac{1}{2 T}\right]$ by defining an auxiliary function

$$
\bar{\Phi}(f)=\sum_{k=0}^{n} p_{k} \cos (k 2 \pi T f)=\gamma-\Phi_{w w}(f),
$$

accordingly $p_{0}=\gamma-r_{0}$ and $p_{n}=-2 r_{n}$ for $n=1, \ldots, N-1$. So the example constraint can be expressed as follows:

$$
\Phi_{w w}(f) \leq \gamma \quad \text { for } f \in\left[\frac{\alpha}{2 \pi T}, \frac{1}{2 T}\right]
$$

if two positive semidefinite, real symmetric matrices $\mathbf{X}, \mathbf{Z}$ exist, for which

$$
\begin{aligned}
\mathbf{L}^{*}(\mathbf{X})+\mathbf{\Lambda}^{*}(\mathbf{Z}, \alpha, 2 \pi-\alpha) & = \\
& \left(\gamma-r_{0},-2 r_{1}, \ldots,-2 r_{N-1}\right) .
\end{aligned}
$$

Since $\mathbf{L}^{*}$ and $\boldsymbol{\Lambda}^{*}$ are linear operators, eq. (19) only expresses linear constraints on the elements of some positive semidefinite matrices. This can be solved efficiently by the already mentioned optimization package [10]. 


\section{Non-constant Upper Bounds}

By using a different auxiliary function, non-constant upper bounds $\Gamma(f)$ can be implemented. This auxiliary function is now defined as:

$$
\bar{\Phi}(f)=\sum_{k=0}^{n} p_{k} \cos (k 2 \pi T f)=\Gamma(f)-\Phi_{w w}(f),
$$

with $p_{0}=\gamma_{0}-r_{0}$ and $p_{n}=\gamma_{n}-2 r_{n}$ for $n=1, \ldots, N-1$, whereby $\gamma_{n}$ is the Fourier series expansion of $\Gamma(f)$. This results in:

$$
\Phi_{w w}(f) \leq \Gamma(f) \text { for } f \in\left[\frac{\alpha}{2 \pi T}, \frac{1}{2 T}\right],
$$

if two positive semidefinite, real symmetric matrices $\mathbf{X}, \mathbf{Z}$ exist, for which

$$
\begin{aligned}
\mathbf{L}^{*}(\mathbf{X})+\boldsymbol{\Lambda}^{*}(\mathbf{Z}, \alpha, 2 \pi-\alpha)= & \\
& \left(\gamma_{0}-r_{0}, \gamma_{1}-2 r_{1}, \ldots, \gamma_{N-1}-2 r_{N-1}\right)
\end{aligned}
$$

So $\Gamma(f)$ can define any upper bound that can be represented as $N$ terms of a Fourier series expansion and covers an interval of the form $\left[\frac{\alpha}{2 \pi T}, \frac{1}{2 T}\right]$ for arbitrary $\alpha$. Using eqs. (14) and (16) intervals of complimentary shape $\left[0, \frac{\alpha}{2 \pi T}\right]$ can be used as well.

\section{Finding appropriate Upper Bounds}

It might seem possible to use only one constraint to represent the whole spectral mask. Although the function $\Gamma(f)$ can approximate any spectral mask $S(f)$ which might serve as a constraint, the approximation is limited by the properties of the Fourier series expansion. This is especially problematic at discontinuities which lead to the Gibbs Phenomenon. Since $\Phi_{w w}(f) \geq 0 \forall f$ is an implicit constraint when working with the autocorrelation coefficients $r_{n}$, any negative value in an upper bound would make no solution possible.

So instead functions are defined, which serve as piecewise continuous upper bounds. When approximating only part of a given spectral mask $S(f)$ or $S(f) /|Q(f)|^{2}$ the Fourier series expansion can't be used because the cosine functions are not orthogonal on any interval $[\alpha, \beta]$. Instead minimizing the squared error for the base function system $\varphi_{n}(f)=\cos (2 \pi n T f)$ on some particular interval,

$$
\min _{\gamma_{n}} \int_{\alpha}^{\beta}\left|S(f)-\sum_{n=0}^{N-1} \gamma_{n} \varphi_{n}(f)\right|^{2} d f
$$

leads to solving a linear equation system. This is equivalent to orthogonalizing the autocorrelation matrix of the base functions on this interval and comes out to:

$$
\sum_{n=0}^{N-1} \gamma_{n} \int_{\alpha}^{\beta} \varphi_{r}(f) \varphi_{n}(f) d f=\int_{\alpha}^{\beta} S(f) \varphi_{r}(f) d f .
$$

Very good approximations of any piecewise continuous function serving as an upper bound can be achieved this way.

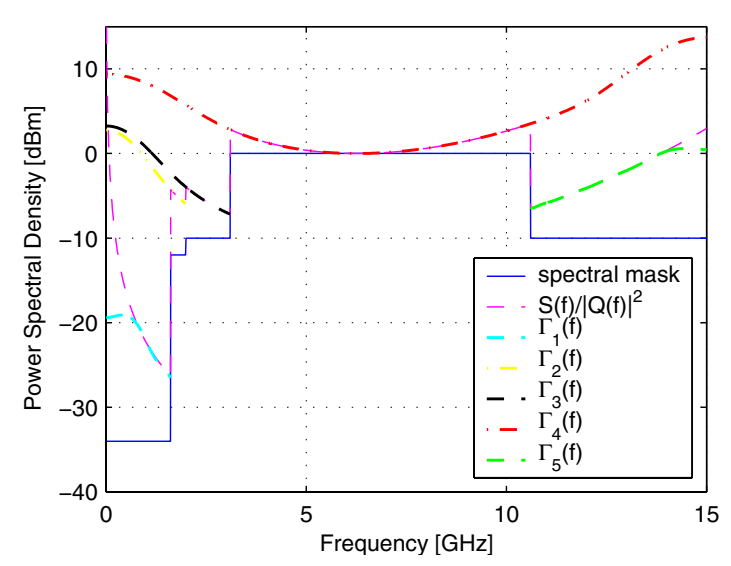

Figure 3: Newly implemented non-constant upper bounds

\section{IMPLEMENTATION}

The interval $[0,15.0] \mathrm{GHz}$ covers the biggest part of the FCC spectral mask $S(f)$. To implement the spectral mask as piecewise continuous constraints without any simplifications, we will have to use five constraints (see Fig. 3):

$$
\begin{array}{ll}
\Phi_{w w}(f) \leq \Gamma_{1}(f) & f \in[0,1.61] \mathrm{GHz} \\
\Phi_{w w}(f) \leq \Gamma_{2}(f) & f \in[0,1.99] \mathrm{GHz} \\
\Phi_{w w}(f) \leq \Gamma_{3}(f) & f \in[0,3.1] \mathrm{GHz} \\
\Phi_{w w}(f) \leq \Gamma_{4}(f) & f \in[0,12.5] \mathrm{GHz} \\
\Phi_{w w}(f) \leq \Gamma_{5}(f) & f \in[10.6,15.0] \mathrm{GHz}
\end{array}
$$

and those constraints are represented by their respective coefficients $\Gamma_{i}(f)=\sum_{k=0}^{N-1} \gamma_{k}{ }^{(i)} \cos (2 \pi k T f)$, calculated through eq. (24). The two possible interval types for the upper bounds, either start at $0 \mathrm{GHz}$ or end at $15.0 \mathrm{GHz}$. Consequently, when using more than two constraints, the upper bounds overlap (see Fig. 3). This can lead to difficulties since the uppers bounds are only calculated dependant on a smaller continuous interval. In fact, no problem arises as long as the discontinuities are positive jumps from one side or drops on the other side respectively. The constraint in the middle actually even serves as an upper bound on the whole interval.

Even when split up into piecewise-continuous intervals, to receive good approximations, the needed upper bounds cannot have a too high gradient. Especially when dividing by the PSD of the Gaussian monocycle, the gradient can become very high in $\Gamma_{1}(f)$ and $\Gamma_{5}(f)$. To avoid poor approximations, $S(f) /|Q(f)|^{2}$ has to be limited, most easily accomplished by cutting off values, e.g., when values in an interval reach a certain multiple of the smallest value. In Fig. 3 values were cut off when $6 \mathrm{~dB}$ above the smallest value of their interval.

\section{DESIGN EXAMPLE}

Fig. 4 shows a design example of $\Phi_{w w}(f)$. It can be seen how $\Phi_{w w}(f)$ approaches $S(f) /|Q(f)|^{2}$ very well (results are plotted for different values of $N)$. $\Phi_{w w}(f)$ is actually above $S(f)$ before being multiplied with $|Q(f)|^{2}$, but this just shows 


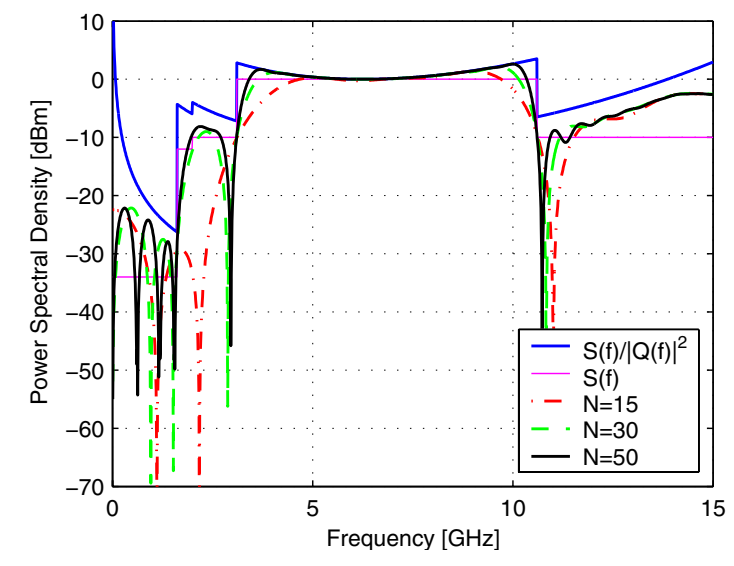

Figure 4: Optimized PSD with non-constant upper bounds

to which extent the allowed energy radiation had not been exploited before by assuming the Gaussian monocycle to be constant.

\begin{tabular}{c|ccc}
$\mathrm{N}$ & 15 & 30 & 50 \\
\hline$\eta_{3}$ adapted to pulse & 0.723 & 0.842 & 0.888 \\
\hline$\eta_{2}$ due to non-const. pulse & 0.633 & 0.698 & 0.726 \\
\hline gain [dB] & 0.580 & 0.811 & 0.877
\end{tabular}

Table 2: Performance of optimization with non-constant pulses

The exact PSD of the waveform $|P(f)|^{2}$ is obtained after multiplication with $|Q(f)|^{2}$ (see Fig. 5). This led to losses before. Now, due to using the extended definition of the upper bounds, results after multiplication fit the FCC spectral mask perfectly and exploit it optimally. For rising pulse train length $N$, results for $\eta$ approach unity (see $\eta_{3}$ in Tab. 2).

The gain in signal power compared to the design of the original linear pulseshaping problem is between $15 \%$ and $20 \%$, which is between $0.6 \mathrm{~dB}$ and $0.9 \mathrm{~dB}$. It should be pointed out that this gain does not require any additional resources in implementation. For the same pulse train length $N$, the gain is achieved solely by equating more optimal coefficients $w_{n}$.

\section{CONCLUSION}

Designing UWB signals using FIR prefiltering can achieve optimal solutions and account for real basic pulse PSDs. It is not necessary to assume the basic pulse PSD as constant and losses due to this assumption can be evaded by incorporating the pulse PSD into the design.

We have achieved a design which gains about $1 \mathrm{~dB}$ of signal power compared to designs disregarding the exact basic pulse PSD. This does not need any extra implementation complexity, since it uses the same filter length $N$. The performance increase is only due to choosing more optimal filter coefficients.

\section{REFERENCES}

[1] M. Z. Win and R. A. Scholtz, "Impulse radio: How it works," IEEE Communications Letter, vol. 2, no. 2, pp. 36-38, Feb. 1998.

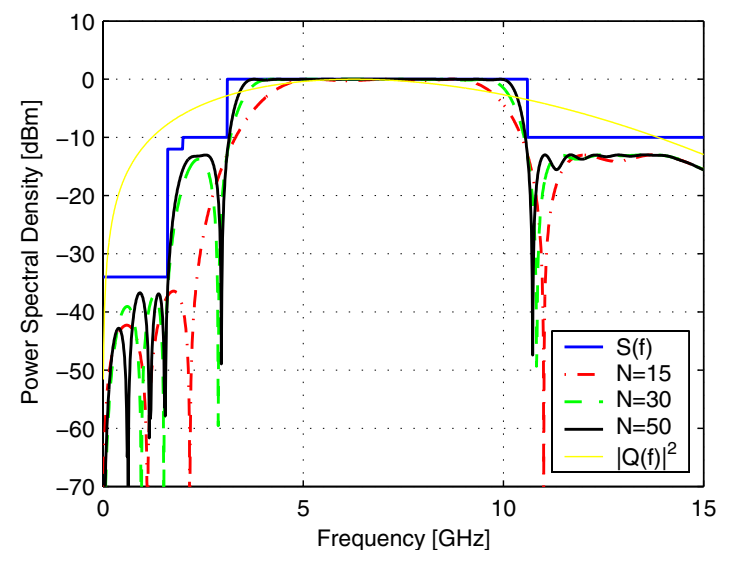

Figure 5: PSD optimized using non-constant upper bounds after multiplication with pulse PSD shows a gain in signal power of about $1 \mathrm{~dB}$

[2] Federal Communications Commission, "In the matter of revision of part 15 of the commission's rules regarding ultra-wideband transmission systems," FCC," First Report and Order, Apr. 2002.

[3] X. Luo, L. Yang, and G. B. Giannakis, "Designing opimal pulse-shapers for ultra-wideband radios," Journal of Communications and Networks, vol. 5, no. 4, pp. 344353, Dec. 2003.

[4] X. Wu, Z. Tian, T. N. Davidson, and G. B. Giannakis, "Optimal waveform design for uwb radios," in Proceedings of the International Conference on Acoustics, Speech and Signal Processing, vol. 4, May 2004, pp. 521-524.

[5] L. Yang and G. B. Giannakis, "Ultra-wideband communications," IEEE Signal Processing Magazine, pp. 26-54, Nov. 2004.

[6] M. Z. Win, "Spectral density of random UWB signals," IEEE Communications Letters, vol. 6, no. 12, pp. 526528, Dec. 2002.

[7] — - "A unified spectal analysis of generalized timehopping spread-spectrum signals in the presence of timing jitter," IEEE Journal on Selected Areas in Communications, vol. 20, no. 9, pp. 1664-1676, Dec. 2002.

[8] B. Parr, B. L. Cho, K. Wallace, and Z. Ding, "A novel ultra-wideband pulse design algorithm," IEEE Communications Letter, vol. 7, no. 5, pp. 219-221, May 2003.

[9] T. N. Davidson, Z.-Q. Luo, and J. F. Sturm, "Linear matrix inequality formulation of spektral mask constraints with applications to fir filter design," IEEE Transactions on Signal Processing, vol. 50, no. 11, pp. 2702-2715, Nov. 2002.

[10] J. F. Sturm, "Using sedumi 1.02, a matlab toolbox for optimization over symmetric cones," Optimization Methods and Software, vol. 11-12, pp. 625-653, 1999. 